\title{
Staff Perspectives on Primary Care Teams as De Facto "Hubs" for Care Coordination in VA: a Qualitative Study
}

Tanya T. Olmos-Ochoa, PhD, MPH' , Purnima Bharath, $M A^{7}$, David A. Ganz, MD, PhD ${ }^{7,2}$, Susan E. Stockdale, $P h D^{1,5}$, Alissa Simon, $M A^{7}$, and Erin P. Finley, $P h D, M P H^{1,3,4}$

'HSR\&D Center for the Study of Healthcare Innovation, Implementation and Policy (CSHIIP), VA Greater Los Angeles Healthcare System, North Hills, CA, USA; ${ }^{2}$ David Geffen School of Medicine, University of California at Los Angeles, Los Angeles, CA, USA; ${ }^{3}$ Veterans Evidence-based Research Dissemination and Implementation Center (VERDICD), South Texas VA Health Care System, San Antonio, TX, USA; ${ }^{4}$ University of Texas Health Science Center at San Antonio, San Antonio, TX, USA; ${ }^{5}$ Department of Psychiatry and Biobehavioral Sciences, University of California, Los Angeles, CA, USA.

BACKGROUND: Improving care coordination is a key priority for many healthcare systems. However, initiatives to improve care coordination are complex to implement and have produced mixed results. A better understanding of how to craft and support implementation of effective care coordination strategies is needed.

OBJECTIVE: To identify and understand the challenges and factors encountered by Patient-Aligned Care Team (PACT) staff in performing care coordination tasks in outpatient clinics in the Veterans Health Administration (VA).

DESIGN: Qualitative study using semi-structured formative evaluation interviews.

PARTICIPANTS: Fourteen interviews with 18 clinical frontline managers and staff from 12 clinic sites across five VA health systems.

INTERVENTIONS: This paper reports on baseline data collected for the Coordination Toolkit and Coaching (CTAC) project. CTAC aims to improve patients' experience of care coordination within VA primary care and between PACT and other outpatient and community settings.

APPROACH: We conducted pre-implementation telephone interviews with frontline managers and staff, primarily nurse managers.

KEY RESULTS: PACT staff described challenges in aligning care coordination priorities across different levels of the VA system, including staff, patients, and leadership. Additionally, PACT staff noted challenges coordinating care both within and outside the VA, and identified resource barriers impeding their care coordination efforts. To address these challenges, staff made several recommendations for improvement, including (1) contingency staffing to address staff burnout; (2) additional PACT training for new staff; (3) clarification of care coordination roles and responsibilities; and (4) and care coordination initiatives that align both with centrally initiated care coordination programs and frontline needs.

CONCLUSION: In the VA and similarly complex healthcare systems, our findings suggest the need for care

NIH trial registry number: NCT03063294

Electronic supplementary material The online version of this article (https://doi.org/10.1007/s11606-019-04967-y) contains supplementary material, which is available to authorized users.

Published online April 22, 2019 coordination strategies that are buttressed by a systemlevel vision for care coordination, backed up by clear roles and responsibilities for information exchange between primary care staff and other settings, and multidimensional accountability metrics that encompass patient-, staff-, and system-level goals.

KEY WORDS: primary care; healthcare delivery; qualitative research; veterans; implementation research.

J Gen Intern Med 34(Suppl 1):S82-S9 DOI: $10.1007 / \mathrm{s} 11606-019-04967-y$

(c) Society of General Internal Medicine (This is a U.S. government work and not under copyright protection in the U.S.; foreign copyright protection may apply) 2019

\section{INTRODUCTION}

Care coordination is a critical component of effective healthcare delivery, ${ }^{1,2}$ and may be defined as deliberate interactions between multiple participants to facilitate appropriate patient care, through information exchange and an understanding of the roles and resources of participants involved. ${ }^{3}$ Care coordination is a nationally recognized priority for healthcare systems, ${ }^{4}$ including the Veterans Health Administration (VA), ${ }^{5}$ with the potential to reduce waste and improve patient outcomes. ${ }^{4}$ However, care coordination initiatives are complex to implement and have achieved mixed results, with interventions employing comprehensive care coordination strategies seeing modest effects on patient health outcomes and patient satisfaction. ${ }^{6}$ To achieve meaningful and sustained improvement in care coordination, we need a better understanding of how to craft and support implementation of effective care coordination strategies.

In 2010, the VA adopted the patient-centered medical home (PCMH) model and implemented Patient-Aligned Care Teams (PACT) - typically composed of a primary care provider, a nurse care manager, a clinical associate (e.g., licensed practical/vocational nurse), and an administrative clerk- to deliver the prevention-focused, team-based patient-centered care that is PCMH's hallmark. ${ }^{7,}{ }^{8}$ Care coordination is a fundamental pillar of PACT implementation. ${ }^{8-10}$ Veterans experience a high burden of chronic disease and mental illness, 
necessitating successful coordination within PACT and between PACT and specialty care providers. ${ }^{11-13}$ Recent initiatives like the Veterans Choice Program and VA MISSION Act of 2018, which aim to augment veterans' access to community care when timely care is unavailable through VA, require increased coordination between VA and community providers. ${ }^{14,}{ }^{15}$ Effective care coordination, however, requires appropriate resources. ${ }^{16}$ In the VA, inadequate staffing and time constraints are well-documented challenges of PACT implementation, ${ }^{17-20}$ often associated with higher staff burnout $^{18}$ and lower scores on PACT implementation metrics. ${ }^{19}$ Competing care delivery priorities and lack of communication from leadership about PACT performance metrics are additional challenges. ${ }^{19}$

Although the literature notes these challenges for PACT, less is known about how care coordination occurs in the context of the resource constraints and competing priorities of routine care. Awareness of PACT staff perspectives on care coordination can provide key information to align centrally initiated care coordination initiatives with frontline needs. This qualitative analysis uses data from the Coordination Toolkit and Coaching (CTAC) project to report on how clinical staff perceive PACT care coordination needs and responsibilities, how they view the priority and urgency of care coordination initiatives, and how contextual factors may impact these initiatives' potential success.

\section{METHODS}

\section{Study Design}

CTAC aims to improve patients' experience of care coordination within VA primary care and between VA primary care and other outpatient (including non-VA community) settings. ${ }^{21}$ Offering resources such as an online toolkit ${ }^{22}$ and distance-based coaching, CTAC helps frontline managers and staff engage in quality improvement efforts to address care coordination challenges in their clinic. CTAC cluster-randomized matched pairs of VA clinics from the same parent health system to one of two implementation strategies: a passive strategy of receiving access to the CTAC online toolkit, ${ }^{21}$ versus an active strategy augmenting the toolkit with weekly, distance-based quality improvement coaching. CTAC is currently ongoing; this paper reports on baseline data collected from participating clinics prior to randomization. CTAC was determined to be non-research by the VA Office of Patient Care Services and the VA Greater Los Angeles Healthcare System's Institutional Review Board.

Clinic recruitment took place between October 2016 and June 2018 using a multilevel approach, including presentations at national and regional teleconferences, and direct outreach to regional and health system leaders and managers. Preliminary clinic eligibility was met if two clinic sites within a facility shared similar panel sizes, numbers of primary care teams, and types of outpatient services offered.

\section{Data Collection}

For each clinic within a matched pair, we asked facility leaders to provide contact information for at least one frontline manager or staff member with detailed knowledge of the clinic. We then invited frontline managers and staff to participate in a semi-structured interview. Interviews (conducted by DG, TO, and $\mathrm{NC}$ ) occurred by phone and were audio recorded, ranging from 30 to $60 \mathrm{~min}$ in length. Although interviews were scheduled with individual frontline staff, in four of the 14 interviews the main participant invited additional frontline staff to join the call. Appropriate confidentiality assurances were provided at the start of each interview.

\section{Data Source}

Guided by formative evaluation principles, ${ }^{23}$ our team created a pre-implementation interview guide to assess potential influences and challenges frontline managers and staff might encounter during implementation of a care coordination improvement effort through participation in CTAC. Questions focused broadly on formative evaluation domains, like understanding the local implementation setting (e.g., clinic site staffing, patient population needs, prior implementation experience, available resources) and identifying potential implementation challenges (e.g., needed resources, staff resistance to adopt new processes, leadership buy-in). The interview guide was pre-tested with a pilot site and refined prior to use in the project (see Supplementary Material).

\section{Data Analysis}

Interviews were transcribed verbatim. Using rapid qualitative methods for inductive analysis, ${ }^{24-26} \mathrm{TO}$ and $\mathrm{PB}$ created sitespecific profiles for the first four transcripts and met with $\mathrm{EF}$, a medical anthropologist, to discuss initial themes. TO and PB then generated structured summaries for each transcript, including the first four, which were reviewed by TO, PB, and EF during group meetings to produce a preliminary code list that was iteratively refined. Transcripts were uploaded to Atlas.ti (version $8, \mathrm{GmbH}$, Berlin) and coded using content analysis principles, ${ }^{27,}{ }^{28}$ with discrepancies in coding resolved through group consensus (TO, PB, and EF). Following coding, general themes were identified and presented to the broader team for discussion and further refinement.

\section{RESULTS}

We completed 14 interviews with 18 clinical frontline managers and staff from 12 clinic sites across five VA health systems in 2017 and 2018. Table 1 describes key site characteristics. We interviewed 16 registered nurses (RN) working in different PACT positions (e.g., nurses 
with a management role-nurse managers; nurses without a management role-PACT nurses) and two physician managers. Frontline managers and staff had been in their current roles an average of 5 years (ranging from 1 to 14 years) at the time of the interviews.

Four overarching themes emerged from the interviews: (1) challenges in aligning priorities for care coordination across staff, patients, and leadership; (2) specific core challenges associated with PACT care coordination (a) within and (b) outside of VA; (3) resource barriers impeding staff's care coordination efforts; and (4) frontline recommendations for improving care coordination.

\section{Aligning Priorities in Care Coordination Across Staff, Patients, and Leadership}

Overall, staff concurred that care coordination was a fundamental part of their role and essential to effective patient care:

"I think care coordination is really what we do. That's my job really. I mean they come in and if there's an issue, I try to assess what the issue is. If I need to ask a specialist or get an X-ray or get labs, then I have to do that...and help the patient figure out how to take care of their problem." [009, Physician Manager]Staff performed care coordination activities daily, with nurses serving as the main "hub" for care coordination between PACT and other VA departments, and between PACT and community providers.

While universally acknowledging the centrality of care coordination to PACT care delivery, staff also described how care coordination activities could interfere with other patient care responsibilities. Tasks such as connecting with patients to remind them about upcoming appointments, following up with discharged patients, ensuring providers were timely in placing orders, reminding patients to schedule their specialty consults, and conveying needed information from specialty and community providers to patients and VA providers were described as taxing. The time required to perform these tasks was viewed as adversely impacting other clinical activities. For example, one manager explained how burdensome daily tasks competed with chronic disease management and were detrimental to the professional development of PACT nurses:

"There's a lot of day-to-day stuff that still gets put on [the RN's] plate, but the real chronic disease part of it does not get priority... as a consequence... their skill set doesn't really get developed either, because they're basically just trying to bring people back in or get them on the phone." [004, Nurse Manager]Staff also perceived the performance metrics and mandates prioritized by VA leadership to be discordant with the priorities of their patients. One nurse summarized how daily 
efforts to meet performance metrics and coordinate care often competed with patients' priorities, resulting in staff feeling overwhelmed and needing direction about what to prioritize:

"It's just more and more they're asking us to do more things...you need to tell me what's the most important thing that you want me to do because I can't do it all ... you already want me to call all of my patients to make sure that they're prepared for their appointment. You want me to call and scrub [the schedule for] patients who may not necessarily need or want the appointment that they were scheduled for. You want me to do the post-hospital phone calls. You want me to check on the patients and then do all of their reminders including their vaccinations, their foot checks... Never mind the fact the patient wants me to hear what he's saying. Those aren't his priorities!" [012, PACT Nurse]As a result of this perceived misalignment, staff members sometimes struggled to define care coordination and to articulate where care coordination fits within the larger set of VA priorities. Although staff members understood that the impetus for care coordination originated from a centrally initiated strategy, they were less clear about how to implement care coordination day to day. One nurse shared how care coordination came to be a clinic priority:

"I think it came from national. They were like, 'you're supposed to be doing this care coordination.'... it was kind of thrust upon everybody in a, 'this was supposed to be done yesterday' kind of movement... it's still an ongoing issue as to what they want us to be doing and how they want us to be doing it." [012, PACT Nurse]Staff's lack of clarity also extended to how care coordination should be prioritized vis-à-vis overall care delivery goals. Staff expressed frustration at misaligned goals, with staff prioritizing some aspects of care delivery while leadership was perceived to prioritize others. One nurse explained the misalignment in goals at different levels within the VA and the lack of appropriate metrics:

"I think [care coordination] is a ten, but...I think administration thinks access is a ten... what we think is a ten and what we think is important for how we do our jobs is not necessarily what national and administration are saying. So, I think the measures that they released are not necessarily measures that are indicative of our care coordination...." [001, Nurse Manager $]$ Staff were most clear and in agreement about their struggles to achieve care coordination on a daily basis within the larger VA context. They described feeling "stretched," "overwhelmed," and like "everything is a priority." One nurse explained:

"...the VA swings constantly, just the newest and the latest thing that's coming down that you have to make sure happens...the bottom line at this point is we have more responsibilities than we have time in the day...If you want to actually define care coordination... a lot of what we do is we push the system so that the individual patient gets what they need...I think the Greek guy's name is Sisyphus, [he] pushes the big rock... gets to the top and then it rolls over. That's us on a daily basis." [001, PACT Nurse]

\section{Core Challenges in PACT Care Coordination}

Within VA. Although care coordination is necessary and challenging for all patients, staff perceived coordinating care for patients who "walk in" to VA clinics without a scheduled appointment as especially difficult. PACTs are required to provide same-day care for walk-in patients with urgent needs. However, patients often lack knowledge about what constitutes an urgent need and about non-face-to-face alternatives (e.g., arranging for medication refills by phone). Staff's uncertainty about which walk-in requests to prioritize and the lack of protected time to perform care coordination tasks resulted in unnecessary disruptions. One nurse explained how staff can inadvertently encourage walk-ins and impact care coordination broadly:

"A patient will come up [to the clerk] and say, 'I'd like to see my provider,' and the first response is, 'You want to walk-in today?' Who's going to say no to that? ... [it] takes up some of our slots [where] I could be getting a little extra PACT care coordination." [011, PACT Nurse]In addition to the challenge posed by walk-ins, staff described how difficult it can be to engage in improvement activities within PACTs, given that providers, nurses, and clerks have different supervisors. One provider expressed frustration with trying to make changes within PACT that might improve care coordination and team functioning:

"I'm not in charge of the clerks, so I can't tell a clerk I need you to do this... I can ask them...but I can't truly tell them...[Their] supervisor tells them to do things a certain way...I can work with them and call their supervisor...but it's complicated." [009, Physician Manager]PACT interactions with other VA departments within their facilities were also complicated, mirroring issues within PACTs. Staff explained how undefined roles created uncertainty about whether specialty or primary care providers were responsible for 
various aspects of patient care. One nurse explained how specialty care providers frequently refer patients back to primary care without appropriate coordination, further burdening PACTs:

"I feel like everything falls back to primary care. [Specialty's] like, 'oh, you were sent over for your nails to be trimmed but you've got this ulcer. You're going to have to go to primary care.' [The patients] just come over, they sign-in as a walk-in, and they're like, 'well [specialty] told us that we needed to have this taken care of and that the treatment had to start in primary care'... Nobody gave [PACT] a heads up...the lines of communication aren't going both ways with specialty and with primary care." [012, PACT Nurse]Staff also highlighted how communication between VA departments could be difficult, with PACT and specialty staff often unaware of each other's processes and operating procedures, leading to duplicated efforts and delays in care. One nurse described not knowing whom to contact in specialty departments:

"One of the problems we have...is getting in contact with specialty care so that patients get scheduled for appointments...You can call several times, you can leave [a] message, and they don't always return calls in a timely manner...the specialty clinics [also] have [staff] turnover and that information isn't widely distributed. So, you're trying to call someone who's left the facility or transferred to another position." [007, Nurse Manager]

Outside VA. When discussing interactions with community entities, staff largely focused on care coordination issues concerning the Veterans' Choice Program, often referred to simply as "Choice." To a lesser extent, they also mentioned coordinating care for dual users of VA benefits and other insurances (e.g., Medicare, private policy). In both cases, care coordination challenges arose in contacting community entities in a timely manner, gaining access to patient data needed to make clinical decisions on patients' behalf (e.g., results of tests performed at a community site), and finding VA formulary medications equivalent to those prescribed by community providers. One nurse explained the issues with Choice as follows:

“...the Choice program, its intent is good, but the way it's managed and maintained and updated is very difficult for the nurses to help patients coordinate their care through it. Even patients who are Vets and work in our clinic that have used Choice and know the system, and know the players, and know who they can call specifically to get things done, they struggle...to the point that they've given up on Choice..." [004, Nurse Manager]Choice was viewed as a frustrating program that fragmented care and made care coordination more difficult, providing few benefits to patients while creating additional workload for PACT members.

\section{Resource Barriers to Care Coordination}

Frontline staff cited gaps in a variety of key resources as an important barrier to care coordination, with staffing vacancies, turnover, and burnout chief among these.

"I think [care coordination] is a great idea, but it's kind of hard to implement new stuff when you don't have a full staff." [005, Nurse Manager]Resource gaps were described as having implications for how care coordination activities were prioritized and delivered. Not having fully staffed PACTs consistently resulted in staff having to cross-cover for other PACTs in their facilities. For some, cross-coverage resulted in long assignments spanning several months to years while awaiting new hires. Having to balance the workload of two or more PACTs over an extended period led staff to report burnout and low morale. One nurse linked burnout with the high turnover among their team's front desk clerks, stating:

"They're all chronically covering a great workload, which again, burns people out and makes them want to leave, and [staffing] is constantly in this death spiral." [004, Nurse Manager]Other factors perceived to impact staffing included non-competitive pay, although this varied by geographic region, and dissatisfaction with the bureaucratic nature of the VA. Time constraints and lack of physical space to co-locate members of the same PACT were also cited as barriers to effective care coordination.

\section{Frontline Recommendations for Improving Care Coordination}

PACT managers and staff identified target areas for care coordination improvements including increasing contingency staffing, clarifying care coordination roles and responsibilities, and more fully aligning care coordination metrics.

Contingency Staffing. Although staff proposed additional hiring to address gaps, they also made specific suggestions for how additional staff might be assigned. Specifically, staff suggested having full-time "floaters" or a "pool" of designated staff who could cover absences on any team for extended periods of time (if necessary), rather than being assigned to their own PACT. Additionally, staff recommended better planning for staff transitions such as retirement, to ensure no lapse in staffing for anticipated staffing changes. 
Clarifying Care Coordination Roles and Responsibilities. PACT staff also highlighted that care coordination tasks overwhelmingly fell on PACT nurses even when other staff (e.g., specialty nurses) were perceived as well or better equipped to handle those requests. In making recommendations for how to better define care coordination roles and responsibilities within VA and across departments, PACT staff also considered the impact on patient care and patient experience. One nurse recommended:

"I think surgical call backs should be done by a surgical case manager. Mental health should be done by mental health. Rather, we [PACT] do 15 calls a day... the patient would benefit from hearing directly from the people who just took care of them...like the surgeon, to make sure that everything's okay. It may be in addition to us. Maybe they should call the first 48 [hours] and maybe we could call at 96 [hours] to make sure they're still doing okay once they've been home for several days." [003, Nurse Manager]In addition, staff highlighted that new staff were typically onboarded with less training on the PACT model than staff trained as part of PACT implementation. The discrepancy in training was most obvious and problematic when defining roles and responsibilities among PACT members. This resulted in nurses with better PACT training taking on more of the work for their teams to the detriment of care coordination and other efforts.

Fully Aligned Care Coordination Metrics. Staff often perceived that performance metrics were misaligned with their day-to-day workflow and patients' needs, with one nurse explaining that PACTs "have all these scattered measures" to address. They called for performance metrics that are more fully aligned with clinic and national priorities around shared goals, helping to focus clinic activities on the priorities national leadership seeks to improve.

\section{DISCUSSION}

Frontline primary care managers and staff in the VA described significant challenges in their efforts to coordinate patient care activities within and outside VA. Although frontline staff believed that care coordination is an essential part of patientcentered care, executing care coordination tasks remained a challenge. Staff expressed uncertainty about how to organize and prioritize care coordination activities on a day-to-day basis, particularly given the perceived lack of alignment between patient, staff, and VA leadership priorities, and the inherent challenge of navigating competing priorities within a resource-constrained environment.

At the outset of PACT implementation in 2010, the VA outlined a vision in which coordination would be achieved "through active interdisciplinary collaboration that is facilitated by registries, information technology, health information exchange and other means" to ensure appropriate and effective care delivery. ${ }^{33}$ As one of the four pillars of PACT (partnerships with veterans, access to care, care coordination, and team-based care), care coordination was further defined as "collaborative" and focused on "building trusted, personal relationships" between PACT members and other providers to coordinate patient care. ${ }^{5,7}$

In contrast to the original vision, this study suggests that PACT frontline staff have not been experiencing care coordination as collaborative and relational. Instead, staff described a lack of clarity about who is responsible for accomplishing specific tasks, a feeling that PACTs are bearing more than their fair share of the coordination workload, and a sense of misalignment between priorities at the staff, patient, and leadership levels. Staff described care coordination as occurring largely in response to PACT performance metrics perceived as fragmented and in competition with other VA priorities (e.g., increasing access to care).

As a result, staff struggled to define and prioritize care coordination in relation to a VA vision. Instead, staff focused on providing examples of care coordination breakdowns and resource challenges to describe their frustrations with coordinating care within and outside the VA. The disconnect between what frontline staff envisioned as effective care coordination (e.g., long-term chronic disease management), the care coordination activities they are able to perform (e.g., 48$\mathrm{h}$ post-discharge follow-up calls), and top-down dissemination of performance metrics that ultimately determine day-to-day prioritization of tasks may make it more challenging for staff to develop a sense of ownership over care coordination and to endeavor to improve it. More specific attention to achieving alignment in care coordination priorities across all levels of VA, and ensuring that mandates lay out clear and cohesive guidance for care coordination and delivery, are likely to be important in improving both frontline staff and patients' experiences of care over time.

These data were gathered in the context of preimplementation formative evaluation interviews conducted by the CTAC team, with the goal of ensuring that CTAC's implementation of care coordination initiatives was appropriately tailored to meet the needs of participating clinics. As a result, interview questions focused on existing and anticipated barriers to care coordination. In response to these findings, the CTAC team made several adaptations, including allowing clinics greater flexibility in selecting a care coordination improvement project and, for coached clinics, encouraging them to start with a more manageable project focusing on improving patient experience of care coordination within primary care before tackling a project focused on coordination between primary care and other settings.

Limitations to these findings include the modest number of interviews conducted, although the multiple sites represented 
were diverse in size and geographic location, and the consistency of findings across sites suggests identified themes were robust. These qualitative data cannot speak to the quality of care coordination occurring at these sites, or whether there were significant associations between factors identified as important by participants (e.g., staffing gaps and care coordination burden). All CTAC sites are within the VA network of care, and results may not generalize to other settings. Additionally, although gathering patients' perspectives of care coordination was not within the scope of the current study, future research would benefit from incorporating the patient perspective.

Although CTAC is being conducted within VA, these findings have relevance for understanding care coordination more broadly, particularly in illustrating how difficult it can be to achieve effective care coordination in real-world settings. ${ }^{34}$ Within increasingly complex and specialized healthcare environments, understanding and accounting for the challenges facing frontline staff charged with implementation remain vital. Significant improvement in care coordination requires buttressing a system-level vision with detailed roles and responsibilities for information exchange between PCMH staff and other settings, multidimensional accountability metrics, and integration of care coordination with existing priorities (e.g., access to care).

Acknowledgements: We want to acknowledge and thank Lisa Rubenstein for co-conceiving CTAC and Debbie Delevan for the administrative support she provided on the project.

Corresponding Author: Tanya T. Olmos-Ochoa, PhD, MPH; HSR\&D Center for the Study of Healthcare Innovation, Implementation and Policy (CSHIIP)VA Greater Los Angeles Healthcare System, North Hills, CA, USA (e-mail: Tanya.Olmos@va.gov).

Funders This material is based upon work supported by the Department of Veterans Affairs, Buality Enhancement Research Initiative through a grant to the Care Coordination QUERI Program (QUE 15-276). The views expressed in this article are those of the authors and do not necessarily reflect the position or policy of the Department of Veterans Affairs or the United States government.

\section{Compliance with Ethical Standards:}

Conflict of Interest: The authors declare that they do not have a conflict of interest.

\section{REFERENCES}

1. McDonald KM, Schultz E, Albin L, et al. Care Coordination Measures Atlas Version 4. Rockville, MD: Agency for Healthcare Research and Quality; 2014.

2. Bodenheimer T, Ghorob A, Willard-Grace R, Grumbach $\mathbf{K}$. The 10 building blocks of high-performing primary care. Ann Fam Med. 2014;12(2): 166-71. https://doi.org/10.1370/afm.1616.

3. Schultz EM, McDonald KM. What is care coordination? International Journal of Care Coordination. 2014;17(1-2):5-24. https://doi.org/10. $1177 / 2053435414540615$

4. National Priorities Partnership. Input to the Secretary of Health and Human Services on priority for the National Quality Strategy. Washington, DC: National Quality Forum, 2011
5. Luck J, Bowman C, York L, Midboe A, Taylor T, Gale R, Asch S. Multimethod evaluation of the VA's peer-topeer toolkit for patientcentered medical home implementation. J Gen Intern Med. 2013;29(Suppl 2):S572-8

6. Powell Davies G, Williams AM, Larsen K, Perkins D, Roland M, Harris MF. Coordinating primary health care: an analysis of the outcomes of a systematic review. Med J Aust. 2008;188(8 Suppl):S65-8.

7. Department of Veterans Affairs. Patient Care Services. Patient Aligned Care Team (PACT) Available at: https://www.patientcare.va.gov/primarycare/PACT.asp. Accessed 3 Aug 2018.

8. Rosland AM, Nelson $\mathbf{K}$, Sun $\mathbf{H}$, et al. The patient-centered medical home in the Veterans Health Administration. Am J Manag Care. 2013;19(7):e263-272.

9. Nelson K, Sun H, Dolan E, Maynard C, Beste L, Bryson C, Schectman G, Fihn SD. Elements of the patient-centered medical home associated with health outcomes among veterans: the role of primary care continuity, expanded access, and care coordination. J Ambul Care Manage. 2014;37(4):331-8. https://doi.org/10.1097/JAC. 0000000000000032 .

10. Nelson KM, Helfrich C, Sun H, Hebert PL, Liu CF, Dolan E, Taylor L, Wong E, Maynard C, Hernandez SE, Sanders W, Randall I, Curtis I, Schectman G, Stark R, Fihn SD. Implementation of the patientcentered medical home in the Veterans Health Administration: associations with patient satisfaction, quality of care, staff burnout, and hospital and emergency department use. JAMA Intern Med. 2014;174(8):1350-8. https://doi.org/10.1001/jamainternmed.2014.2488.

11. Kilbourne AM, Hynes D, O'Toole T, Atkins D. A research agenda for care coordination for chronic conditions: aligning implementation, technology, and policy strategies. Transl Behav Med. 2018;8(3):515-521. https://doi.org/10.1093/tbm/ibx084.

12. Balbale SN, Etingen B, Malhiot A, Miskevics S, LaVela SL. Perceptions of chronic illness care among veterans with multiple chronic conditions. Mil Med. 2016;181(5):439-44. https://doi.org/10.7205/MILMED-D-1500207

13. Jordan N, Sohn MW, Bartle B, Valenstein M, Lee Y, Lee TA. Association between chronic illness complexity and receipt of evidence-based depression care. Med Care. 2014;52(Suppl 3):S126-31. https://doi.org/10. 1097/MLR.0000000000000036.

14. Department of Veterans Affairs. Expanded access to non-VA care through the veterans choice program. Final rule. Fed Regist. 2018;83(92):21893-7.

15. Department of Veterans Affairs. Available at: https://veterans.house. gov/uploadedfiles/va_mission_act_summary.pdf. Accessed 20 July 2018.

16. Meyers D, Peikes D, Genevro J, Peterson G, Taylor EF, Tima Lake T, Smith K, Grumback K. The Roles of Patient-Centered Medical Homes and Accountable Care Organizations in Coordinating Patient Care. AHRQ Publication No. 11-M005-EF. Rockville, MD: Agency for Healthcare Research and Quality; 2010. Available at: https://escholarship.org/uc/ item/2997j352.

17. Butler A, Canamucio A, Macpherson D, Skoko J, Gala T. Primary care staff perspectives on a virtual learning collaborative to support medical home implementation. JGIM. 2014;29(suppl 2):S579-88. https://doi. org/10.1007/s11606-013-2668-x.

18. Helfrich CD, Dolan ED, Simonetti J, Reid RJ, Joos S, Wakefield BJ, Schectman G, Stark R, Fihn SD, Harvey HB, Nelson K. Elements of team-based care in a patient-centered medical home are associated with lower burnout among VA primary care employees. JGIM. 2014;29(Suppl 2):S659-66. https://doi.org/10.1007/s11606-013-2702-z.

19. Helfrich CD, Sylling PW, Gale RC, Mohr DC, Stockdale SE, Joos S, Brown EJ, Grembowski D, Asch SM, Fihn SD, Nelson KM, Meredith LS. The facilitators and barriers associated with implementation of a patient-centered medical home in VHA. Implement Sci 2016;11(24): 1-9. https://doi.org/10.1186/s13012-016-0386-6

20. Tuepker A, Kansagara D, Skaperdas E, Nicolaidis C, Joos S, Alperin M, Hickman D. "We've not gotten even close to what we want to do": a qualitative study of early patient-centered medical home implementation. JGIM. 2014;29(Suppl 2):S614-22. https://doi.org/10.1007/s11606013-2690-Z

21. Ganz DA, Barnard JM, Smith NZY, Miake-Lye IM, Delevan DM, Simon A, Rose DE, Stockdale SE, Chang ET, Noël PH, Finley EP, Lee ML, Zulman DM, Cordasco KM, Rubenstein LV. Development of a webbased toolkit to support improvement of care coordination in primary care. Transl Behav Med. 2018;8(3):492-502. https://doi.org/10.1093/ tbm/ibx072. 
22. Ganz DA, Barnard JM. Care Coordination Toolkit. Care coordination toolkit - intranet only. 2006.

23. Stetler CB, Legro MW, Wallace CM et al. The role of formative evaluation in implementation research and the QUERI experience. J Gen Intern Med. 2006;21(Suppl 2):S1-S8.

24. Bernard HR. Research Methods in Anthropology, 5th edition. AltaMira Press. p 7

25. Hamilton A. Cyberseminar: Qualitative methods in rapid turn-around health services research. December 11, 2013. VA HSR\&D cyberseminar spotlight on women's health. http://www.hsrd.research.va.gov/for_ researchers/cyber_seminars/archives/780-notes.pdf.

26. Watkins DC. Rapid and rigorous qualitative data analysis. Int $\mathrm{J}$ Qual Methods. 2017;16:1-9. DOI: https://doi.org/10.1177/1609406917712131

27. Cole FL. Content analysis: process and application. Clin Nurse Spec. 1998;2(1):53-57.

28. Hsiu-Fanh H, Shannon SE. Three approaches to qualitative content analysis. Qual Health Res. 2005;15(9):1277-1288. https://doi.org/10. $1177 / 1049732305276687$

29. Casalino LP, Wu FM, Ryan AM, Copeland K, Rittenhouse DR, Ramsay PP, Shortell SM. Independent practice associations and physicianhospital organizations can improve care management for smaller practices. Health Aff. 2013;32(8):1376-1382. https://doi.org/10.1377/hlthaff. 2013.0205
30. United States Census Bureau. Regions and divisions with state FIPS codes. Available at: https://www2.census.gov/geo/docs/maps-data/ maps/reg_div.txt. Accessed 2 Nov 2018.

31. United Stated Department of Agriculture. Rural-urban commuting area codes. Available at: https://www.ers.usda.gov/data-products/rural-urban-commuting-area-codes.aspx. Accessed 2 Nov 2018.

32. Nelson KM, Helfrich C, Sun H, Hebert PL, Liu C, Dolan E, Taylor L, Wong E, Maynard C, Hernandez SE, Sander W, Randall I, Curtis I, Schectman G, Stark R, Fihn SD. Implementation of the patient-centered medical home in the veterans health administration: associations with patient satisfaction, quality of care, staff burnout, and hospital and emergency department use. J Am Med Assoc Intern Med. 2014;174(8):1350-1358. https://doi.org/ 10.1001/jamainternmed.2014.2488.

33. Patient Centered Primary Care Implementation Work Group. Patient centered medical home model concept paper: veterans health administration primary care services.

34. Taylor EF, Lake T, Nysenbaum J, Peterson G, Meyers D. Coordinating Care in The Medical Neighborhood: Critical Components and Available Mechanisms. White Paper (Prepared by Mathematica Policy Research Under Contract No. HHSA290200900019I TO2). AHRQ Publication No. 11-0064. Rockville, MD: Agency for Healthcare Research and Quality; 2011.

Publisher's Note Springer Nature remains neutral with regard to jurisdictional claims in published maps and institutional affiliations. 